\title{
Retrospective Claims Analysis Indirectly Comparing Medication Adherence and Persistence Between Intravenous Biologics and Oral Small-Molecule Therapies in Inflammatory Bowel Diseases
}

\author{
Kellyn Moran · Kyle Null · Zhongwen Huang • Trevor Lissoos • \\ Sunanda Kane
}

Received: May 24, 2019 / Published online: August 5, 2019

(c) The Author(s) 2019

\section{ABSTRACT}

Introduction: Patients' adherence to and persistence on treatment for inflammatory bowel disease (IBD) can vary, depending on type and distribution of disease and treatment modality. We aim to identify differences in adherence and persistence with treatments with different administration routes (intravenous vs oral) in IBD.

Methods: A retrospective cohort analysis of a claims database of adult patients diagnosed with IBD or rheumatoid arthritis (RA) who began treatment with vedolizumab, tofacitinib, or infliximab from January 2015 through December 2015. Adherence evaluated by proportion of days covered (PDC) and cumulative

Enhanced Digital Features To view enhanced digital features for this article go to https://doi.org/10.6084/ m9.figshare.8938112.

Electronic Supplementary Material The online version of this article (https://doi.org/10.1007/s12325019-01037-x) contains supplementary material, which is available to authorized users.

K. Moran · K. Null · Z. Huang · T. Lissoos

U.S. Medical Affairs, Takeda Pharmaceuticals U.S.A., Inc., Deerfield, IL, USA

S. Kane $(\square)$

Division of Gastroenterology and Hepatology, Mayo

Clinic, Rochester, MN, USA

e-mail: kane.sunanda@mayo.edu days with gaps at least $20 \%$ beyond expected interval (CG20) using multivariable generalized linear equation models. Persistence assessed as time to treatment discontinuation over 12 months of follow-up using Kaplan-Meier estimates and Cox proportional hazards models; proportion of persistent patients determined via multivariable logistic regression. Indirect comparisons across disease states adjusted using infliximab data.

Results: After indirect adjustment by disease, mean PDC difference was significantly higher (difference of $4.7 \% ; P=0.0376$ ) and mean CG20 was lower (difference of 15 days; $P=0.0646$ ) but not statistically significant in vedolizumab/IBD than tofacitinib/RA.

Conclusion: We describe a novel adjustment method for interdisease treatment differences using infliximab treatment patterns to bridge differences between IBD and RA. After adjustment, adherence was higher with infusions than oral medications, which may affect outcomes. Indirect comparisons between vedolizumab and tofacitinib are not generalizable and should be confirmed in tofacitinib-treated IBD patients.

Funding: Takeda Pharmaceuticals U.S.A., Inc.

Keywords: Adherence; Databases; Drug administration; Inflammatory bowel disease; Monoclonal antibodies; Outcomes research/analysis 


\section{INTRODUCTION}

Inflammatory bowel disease (IBD) is a chronic gastrointestinal inflammatory condition that can require lifelong treatment [1]. Adherence to long-term therapy for chronic illnesses is suboptimal, which can lead to increased disease activity, loss of response to therapy, and increased costs of care [1-4]. Adherence to and persistence on treatment for patients with IBD are no exception, and can vary greatly, depending on type and distribution of disease and treatment modality (oral versus infusion versus injection) $[1,5,6]$.

In IBD, biologics are joining non-gut-selective conventional therapies, such as 5-aminosalicylates, corticosteroids, and immunomodulators, in the treatment paradigm [7]. Historically, rates of nonadherence to oral 5-aminosalicylates and thiopurines for IBD are highly variable, ranging from $7 \%$ to $72 \%$, depending on the tools used to measure nonadherence [8]. Nonadherence to biologic medications for patients with IBD has been estimated to be approximately 30\% in Crohn's disease (CD) and 50\% in ulcerative colitis (UC) [6]. Poor adherence in patients with IBD is linked to worse outcomes, including disease flares, disease progression, higher healthcare resource utilization, and immunogenicity [1]. Treatment-related factors such as dose, frequency, and route of administration have been associated with adherence in IBD $[9,10]$. In this context, the introduction of newer oral agents into the IBD treatment armamentarium, with frequent dosing schedules and unsupervised administration, raises questions about the impact administration route could have on adherence to or persistence on therapy [11]. Given the potential adverse effect that suboptimal adherence from the initiation of therapy could have on long-term outcomes, it could be useful to be able to forecast potential adherence issues as soon as possible upon adoption of these agents without having to wait for accumulated real-world data.

Vedolizumab is the only gut-selective agent approved in the USA (in 2014) for the treatment of adults with UC and CD as an intravenous infusion at 0,2 , and 6 weeks and then every
8 weeks [12]. The systemically acting oral smallmolecule JAK inhibitor tofacitinib was approved in the USA for the treatment of rheumatoid arthritis (RA) in 2012 and recently approved for the treatment of moderately to severely active UC (May 2018) [13, 14].

A comparison of adherence and persistence between vedolizumab- and tofacitinib-treated patients could provide insights into the impact of treatment-related factors, such as route of administration and patient medication utilization in IBD. The objective of this study was to compare adherence and persistence for vedolizumab and tofacitinib within 12 months after treatment initiation; our focus was IBD, but we used data from IBD and RA patients for vedolizumab and tofacitinib, respectively. Because tofacitinib was not indicated for UC at the time of this study, we developed methodology that would allow the anticipation of adherence and persistence with tofacitinib treatment based on RA usage patterns. Data on infliximab, an intravenous agent indicated for both IBD and RA [15], provided a framework for the indirect comparison between vedolizumab and tofacitinib to be adjusted for disease-specific differences.

\section{METHODS}

\section{Patients and Study Design}

We performed a retrospective cohort study using the Truven MarketScan ${ }^{\circledR}$ database of medical and pharmacy claims representing approximately 170 million covered lives. Commercially insured or Medicare-insured patients ( $\geq 18$ years old) with IBD or RA who initiated vedolizumab or tofacitinib during January 2015 through December 2015 and those who initiated infliximab for RA or IBD in the USA were eligible for the study (Fig. S1 in the supplementary material). Index date was defined as the date of drug initiation. The study groups were defined by diagnosis (UC, CD, or RA) and index therapy (vedolizumab, infliximab, or tofacitinib).

The primary purpose of this study was to compare adherence to and persistence on oral 
versus infusion medications using vedolizumab and tofacitinib as surrogate treatments for this comparison. However, at the time of the study, these medications were approved in the USA for different disease states. Vedolizumab is a gutselective therapy approved for IBD; tofacitinib is a systemically acting agent approved for RA, and only recently for UC. Therefore, we validated this comparison and accounted for differences in adherence to and persistence on therapies across disease states by including infliximab, a non-gut-selective biologic indicated for the treatment of both IBD and RA. Adherence to and persistence on infliximab in an IBD population were compared with those in an RA population to validate the comparability between vedolizumab and tofacitinib and correct for any potential differences between diseases. The disease state effect on adherence and persistence estimated from the infliximab/IBD and infliximab/RA comparison was assumed to be similar to the effect on the projected adherence and persistence with tofacitinib in IBD. Eligible patients included those with continuous enrollment for 12 months before and after drug initiation, with at least two diagnoses of UC or CD (for vedolizumab or infliximab) or RA (for tofacitinib or infliximab) at least 30 days apart during the baseline period, and who had at least two doses/fills for the index treatment before discontinuation. Patients were excluded if they had a diagnosis of an infliximab-indicated inflammatory condition (ankylosing spondylitis, psoriasis, or psoriatic arthritis). Patients with claims for both IBD (UC or CD) and RA were excluded.

\section{Compliance with Ethics Guidelines}

This study only involved the use of anonymized electronic healthcare records, and researchers did not have any access to named or identifiable patient information. As such, this study does not meet the definition of human subject research as defined in federal regulations 45 CFR 46.102. The study was therefore exempt from institutional review board review.

\section{Study Endpoints}

Adherence over 12 months of follow-up was assessed as (1) mean proportion of days covered (PDC), (2) proportion of patients with PDC $\geq 80 \%$, and (3) cumulative days with a gap at least 20\% beyond the expected interval (CG20). PDC was calculated as the ratio of the number of days covered by the medication divided by the number of days in the measurement period. CG20 was calculated as the summation of days without coverage that were at least $20 \%$ of the previous day's supply. Persistence was assessed as the time to treatment discontinuation and the proportion of patients who did not discontinue treatment over 12 months of follow-up. Discontinuation was defined by a gap in therapy at least 1.5 times the prior day's supply, beginning from the end of the last supply. A sensitivity analysis was conducted with a gap in therapy at least 0.5 times the prior day's supply for a more stringent definition of discontinuation. Expected days' supply for vedolizumab or infliximab infusions was based on labeled dosing (14 days for first infusion, 28 days for second infusion, 56 days after that). Days' supply for tofacitinib was reported on the claim. Patients receiving early refills of tofacitinib were assumed to have finished the prior supply of medication before initiating the new supply. This adjustment was not made for infusion therapies because these patients were not assumed to gain additional days covered from an early infusion.

\section{Statistical Analyses}

Bivariate comparisons among patients treated with vedolizumab versus tofacitinib and infliximab for IBD versus infliximab for RA were conducted using chi-squared tests for categorical variables, two-sample $t$ tests for parametric continuous variables, and Mann-Whitney $U$ test for nonparametric continuous variables. The difference in mean PDC and CG20 between vedolizumab and tofacitinib was estimated using multivariable generalized linear models (GLMs). Covariates analyzed included demographics, health plan type, previous biologic 
treatments, hospitalization or an emergency department (ED) visit during baseline, comorbidity burden, prescription burden, and index therapy cost. A backward selection method was used to select important covariates, using a threshold of $P$ value $<0.10$ for inclusion in the final GLM.

The difference in the proportion of patients with $\mathrm{PDC} \geq 80 \%$ between vedolizumab and tofacitinib was estimated using multivariable logistic regression. Persistence was assessed as time to treatment discontinuation using the Kaplan-Meier survival analysis, log-rank test, and multivariable Cox proportional hazards model; the proportion of persistent patients was determined using a multivariable logistic regression model. The difference in mean PDC and CG20 and the proportion with PDC $\geq 80 \%$ and persistent at 12 months between patients initiating vedolizumab versus tofacitinib was indirectly adjusted by the differences observed in the outcomes among patients initiating infliximab/IBD versus infliximab/RA in the regression models to account for underlying disease differences.

\section{RESULTS}

\section{Baseline Characteristics}

A total of 2634 patients were eligible for this study; $51.6 \%$ had a diagnosis of IBD (UC $42.2 \%$ $[n=573]$; CD 57.8\% [ $n=785])$ and $48.4 \%$ had a diagnosis of RA. When grouped by treatment type, there were 457 vedolizumab/IBD, 901 infliximab/IBD, 378 infliximab/RA, and 898 tofacitinib/RA claims analyzed (Table 1). Demographic and clinical characteristics were identified over the 12 months before the index date and were different between disease states. The mean age was significantly lower in patients with IBD than in patients with RA (approximately 42 and 55 years, respectively). This difference is not unexpected given the different average ages at onset for both diseases. A significantly higher proportion of patients with RA (82.4\%) than IBD (52.4\%) were women. Patients with IBD were significantly more likely to have an ED visit $(17.8 \%$ vs $6.2 \% ; P<0.0001)$ or hospitalization $(22.7 \%$ vs $0.7 \% ; P<0.0001)$ than patients with RA in the year before the index date. Patients with IBD were more likely to be biologic naïve than patients with RA $(57.3 \%$ vs $31.2 \% ; P<0.0001)$. Patients were classified as biologic naive if they had no prior biologic exposure during the pre-index period. Within each disease state, patients initiating infliximab were more likely to be biologic naïve than those initiating vedolizumab for IBD or tofacitinib for RA (Table 1). Comorbidity burden, defined by the Quan-Charlson Comorbidity Index (excluding RA from the list of conditions), and prescription burden (defined by the number of unique medications dispensed within 6 months before the index date) were significantly $(P<0.0001)$ higher in patients with RA than those with IBD.

\section{Adherence}

The unadjusted mean PDC (77.7\% vs $68.2 \%$; $P<0.0001)$ and proportion of patients with PDC $\geq 80 \%$ (61.5\% vs $45.5 \% ; P<0.0001)$ were significantly higher among those who initiated vedolizumab than tofacitinib (Fig. 1a, b). Generally, adherence was higher among patients with IBD than RA, as evidenced by the higher mean PDC (infliximab/IBD 79.6\% vs infliximab/ RA $74.9 \% ; P=0.0038$ ) and proportion with PDC $\geq 80 \%$ (infliximab/IBD $69.0 \%$ vs infliximab/RA $57.9 \% ; \quad P=0.0001)$ among those receiving infliximab for IBD versus RA (Fig. 1a, b), suggesting that there could be some diseaserelated differences in adherence across disease states that were corrected for in the generalized model data provided below.

Crude and multivariable-adjusted GLMs were used to evaluate the difference in mean PDC between those initiating vedolizumab and initiating tofacitinib. The crude least-square mean difference in PDC between vedolizumab and tofacitinib patients was $9.6 \%$ (95\% confidence interval $[\mathrm{CI}] 6.6-12.6 ; P<0.0001)$ and $5.0 \%$ (95\% CI $0.6-9.4 ; P=0.0245$ ) before and after adjustment for the difference between infliximab/IBD and infliximab/RA, respectively (Table S1). 
Table 1 Baseline demographics and clinical characteristics by disease state and initiated treatment

\begin{tabular}{|c|c|c|c|c|c|c|}
\hline & \multirow{2}{*}{$\begin{array}{l}\text { VDZ/IBD } \\
(n=457)\end{array}$} & \multirow{2}{*}{$\begin{array}{l}\text { IFX/IBD } \\
(n=901)\end{array}$} & \multirow{2}{*}{$\begin{array}{l}\text { IFX/RA } \\
(n=378)\end{array}$} & \multirow{2}{*}{$\begin{array}{l}\text { TOF/RA } \\
(n=898)\end{array}$} & \multicolumn{2}{|l|}{$P$ value ${ }^{a}$} \\
\hline & & & & & $\begin{array}{l}\text { VDZ/IBD } \\
\text { vs TOF/RA }\end{array}$ & $\begin{array}{l}\text { IFX/IBD } \\
\text { vs IFX/RA }\end{array}$ \\
\hline Age, mean (SD), years & $44.4(13.9)$ & $41.0(15.5)$ & $55.1(12.5)$ & $55.2(11.3)$ & $<0.0001$ & $<0.0001$ \\
\hline \multicolumn{7}{|l|}{ Sex, $n(\%)$} \\
\hline Male & $219(47.9)$ & $428(47.5)$ & $74(19.6)$ & $151(16.8)$ & \multirow[t]{2}{*}{$<0.0001$} & \multirow[t]{2}{*}{$<0.0001$} \\
\hline Female & $238(52.1)$ & $473(52.5)$ & $304(80.4)$ & $747(83.2)$ & & \\
\hline \multicolumn{7}{|c|}{ Prior resource utilization ${ }^{\mathrm{b}}, n(\%)$} \\
\hline ED visit & $70(15.3)$ & $172(19.1)$ & $37(9.8)$ & $42(4.7)$ & $<0.0001$ & $<0.0001$ \\
\hline Hospitalization & $80(17.5)$ & $228(25.3)$ & $3(0.8)$ & $6(0.7)$ & $<0.0001$ & $<0.0001$ \\
\hline \multicolumn{7}{|c|}{ Number of prior biologics, $n(\%)$} \\
\hline 0 & $155(33.0)$ & $627(69.6)$ & $163(43.1)$ & $235(26.2)$ & \multirow[t]{4}{*}{$<0.0001$} & \multirow[t]{4}{*}{$<0.0001$} \\
\hline 1 & $226(49.5)$ & $245(27.2)$ & $136(36.0)$ & $385(42.9)$ & & \\
\hline 2 & $66(14.4)$ & $27(3.0)$ & $63(16.7)$ & $211(23.5)$ & & \\
\hline 3 & $14(3.1)$ & $2(0.2)$ & $16(4.2)$ & $67(7.5)$ & & \\
\hline \multicolumn{7}{|c|}{ Quan-Charlson Comorbidity Index, $n$ (\%) (excluding RA) } \\
\hline 0 & $321(70.2)$ & $649(72.0)$ & $185(48.9)$ & $476(53.0)$ & \multirow[t]{6}{*}{$<0.0001$} & \multirow[t]{6}{*}{$<0.0001$} \\
\hline 1 & $60(13.1)$ & $120(13.3)$ & $110(29.1)$ & $246(27.4)$ & & \\
\hline 2 & $51(11.2)$ & $93(10.3)$ & $51(13.5)$ & $97(10.8)$ & & \\
\hline 3 & $13(2.8)$ & $24(2.7)$ & $23(6.1)$ & $46(5.1)$ & & \\
\hline 4 & $6(1.3)$ & $8(0.9)$ & $6(1.6)$ & $15(1.7)$ & & \\
\hline$\geq 5$ & $6(1.3)$ & $7(0.8)$ & $3(0.8)$ & $18(2.0)$ & & \\
\hline \multicolumn{7}{|c|}{ Prescription burden ${ }^{c}, n(\%)$} \\
\hline 0 & $28(6.1)$ & $76(8.4)$ & $21(5.6)$ & $21(2.3)$ & \multirow[t]{6}{*}{$<0.0001$} & \multirow[t]{6}{*}{$<0.0001$} \\
\hline 1 & $12(2.6)$ & $43(4.8)$ & $4(1.1)$ & $21(2.3)$ & & \\
\hline 2 & $37(8.1)$ & $75(8.3)$ & $11(2.9)$ & $35(3.9)$ & & \\
\hline 3 & $50(10.9)$ & $99(11.0)$ & $14(3.7)$ & $56(6.2)$ & & \\
\hline 4 & $40(8.8)$ & $92(10.2)$ & $33(8.7)$ & $77(8.6)$ & & \\
\hline$\geq 5$ & $290(63.5)$ & $516(57.3)$ & $295(78.0)$ & $688(76.6)$ & & \\
\hline
\end{tabular}

$E D$ emergency department, IBD inflammatory bowel disease, IFX infliximab, $R A$ rheumatoid arthritis, $S D$ standard deviation, TOF tofacitinib, $V D Z$ vedolizumab

${ }^{a} P$ values are from a chi-squared test for categorical variables, two-sample Student $t$ test for parametric continuous variables, or Wilcoxon-Mann-Whitney $U$ test for nonparametric continuous variables

b Patients hospitalized subsequent to an ED visit within 2 days were counted as having a hospitalization

${ }^{c}$ Prescription burden was calculated as the number of unique prescription medications filled within 180 days before the index date but with available days' supply within the 90 days before the index date. Unique prescription medications are defined as distinct classes of medications according to the therapeutic class 

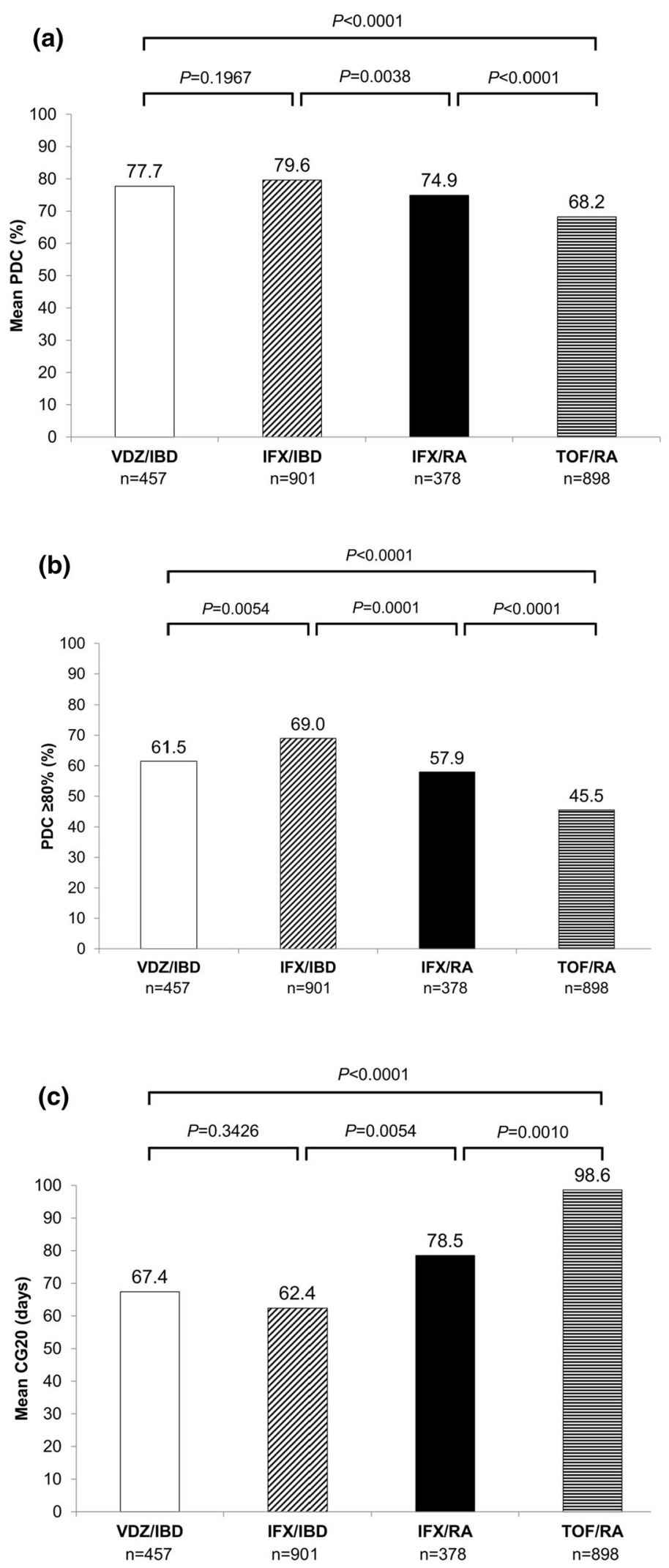
4Fig. 1 Adherence among patients initiating vedolizumab, infliximab, or tofacitinib as measured by a mean PDC, b $\mathrm{PDC} \geq 80 \%$, and c CG20. CG20, cumulative days with gap $\geq 20 \%$ beyond the expected interval. IBD inflammatory bowel disease, IFX infliximab, PDC mean proportion of days covered, RA rheumatoid arthritis, TOF tofacitinib, VDZ vedolizumab

After adjustment for confounders (age, sex, and prior hospitalizations), mean difference in PDC was $10.2 \%$ (95\% CI 6.9-13.4; $P<0.0001)$ higher in the vedolizumab group than in the tofacitinib group $(76.2 \%$ vedolizumab/IBD vs $66.0 \%$ tofacitinib/RA) (Table S1). After indirect adjustment for disease state differences (78.4\% infliximab/IBD vs $72.8 \%$ infliximab/RA), mean difference in PDC was $4.7 \%$ higher in the vedolizumab group than in the tofacitinib group (95\% CI 0.3-9.0; $P=0.0376$ ) (Table S1).

Among patients with IBD, there was no significant difference in unadjusted mean PDC among those treated with vedolizumab versus infliximab (vedolizumab 77.7\%; infliximab 79.6\%; $P=0.1967$ ) (Fig. 1a). After adjustment for confounders, no significant difference in mean PDC between these two groups was observed. The infliximab/RA group had a significantly higher mean PDC than the tofacitinib/RA group (adjusted model mean difference 6.8\%; 95\% CI 3.6-10.0; $P<0.0001$ ) (Table S1), highlighting that mode of administration may impact adherence to medication within the same disease state.

Crude and multivariable-adjusted logistic regression was used to estimate odds of having PDC $\geq 80 \%$ among patients taking vedolizumab versus tofacitinib (Table S2). Compared with tofacitinib-treated patients, vedolizumabtreated patients had approximately two times greater odds of having a PDC $\geq 80 \%$ after adjustment for prior hospitalization (odds ratio [OR] 2.1; 95\% CI 1.6-2.6). After indirect adjustment for the difference between disease states and prior hospitalizations, there was a $4.3 \%$ difference in the proportion of patients with PDC $\geq 80 \%$ among those initiating vedolizumab compared with tofacitinib $(P=0.3782)$. Odds for being adherent were significantly higher for those treated with infliximab for IBD than for RA (OR 1.8; 95\% CI 1.4-2.3) (Table S2), suggesting differences between disease states.

Unadjusted mean CG20 was 67.4 days in the vedolizumab/IBD group and 98.6 days in the tofacitinib/RA group and was significantly lower in patients initiating vedolizumab than tofacitinib $(P<0.0001)$ (Fig. 1c). Similar to PDC, differences in CG20 were observed between patients with IBD and those with RA, suggesting differences in cumulative gaps across disease states. In the crude model, there were on average $32(P<0.0001)$ and $16(P=0.0475)$ fewer gap days in therapy among those on vedolizumab/IBD than tofacitinib/RA before and after adjustment for the difference between infliximab/IBD and infliximab/RA, respectively (Table S1).

Based on a multivariable-adjusted GLM, mean CG20 was significantly lower in the vedolizumab/IBD group than the tofacitinib/RA group (difference of 32.7 days; $P<0.0001$ ) after adjustment for confounders (age, sex, and prior hospitalizations) (Table S1). After accounting for disease state differences, there were on average 15 fewer gap days in therapy among those on vedolizumab/IBD than tofacitinib/RA, but this difference was not statistically significant $(P=0.0646)$ (Table S1). CG20 was significantly lower in the infliximab/IBD group than the infliximab/RA group $(P=0.0062)$ and in the infliximab/RA group than the tofacitinib/RA group $(P=0.0005)$. There was no significant difference in the mean CG20 between the vedolizumab/IBD and infliximab/IBD groups $(P=0.3057)$.

\section{Persistence}

Patients treated with vedolizumab had a significantly longer time to treatment discontinuation than those on tofacitinib (time to $25 \%$ discontinuation 196 days vs 156 days; $P=0.0012$ ) (Fig. 2). Time to $25 \%$ discontinuation was significantly different among all comparison groups (data not shown). Unadjusted percentage of persistent patients was significantly higher in the vedolizumab/IBD group than the tofacitinib/RA group (65.6\% vs $54.9 \%$; $P=0.0001)$ and significantly lower than in the 


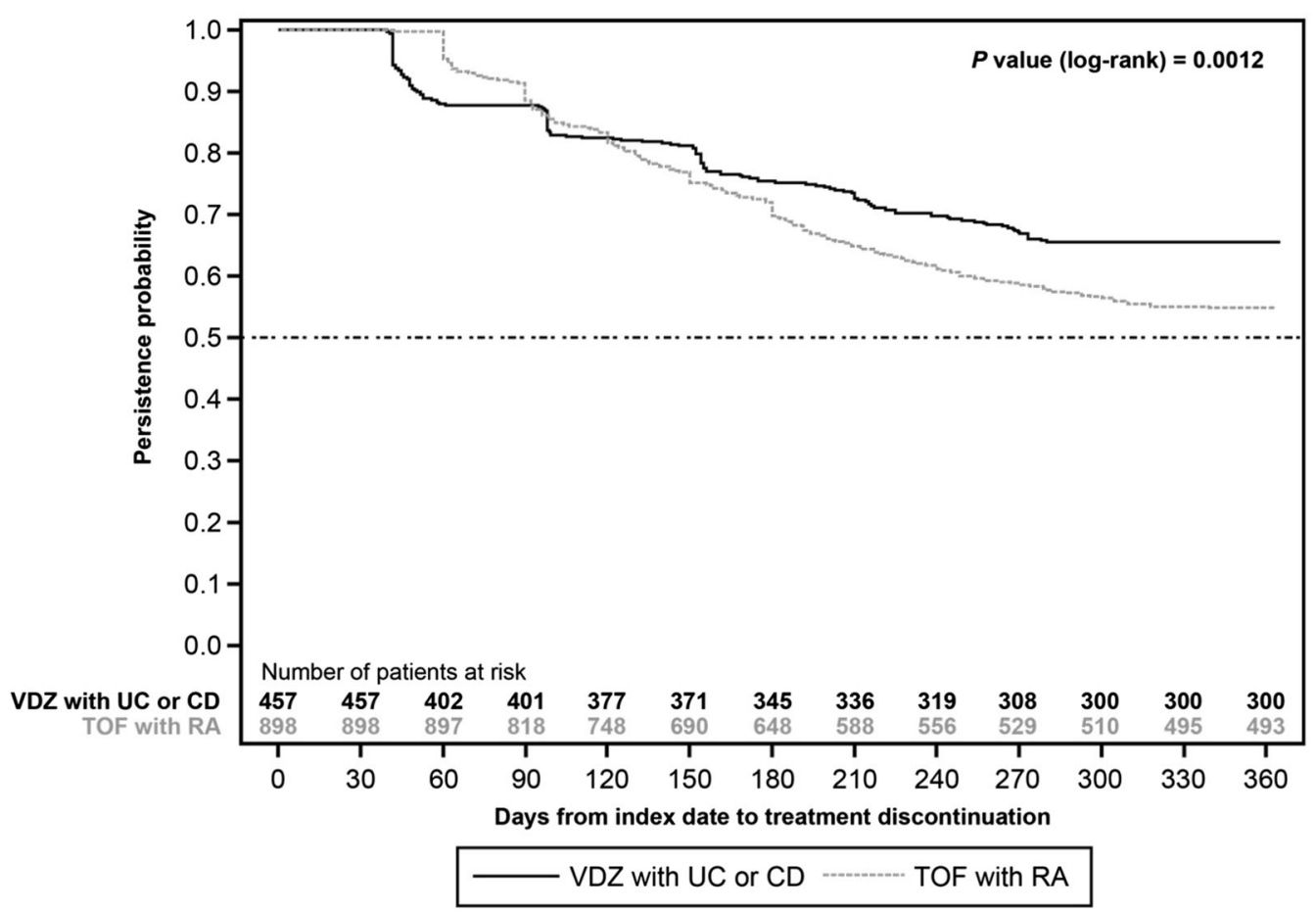

Fig. 2 Kaplan-Meier survival curve for time to treatment discontinuation among patients initiating vedolizumab compared with tofacitinib. CD Crohn's disease, RA rheumatoid arthritis, TOF tofacitinib, UC ulcerative colitis, VDZ vedolizumab

infliximab/IBD group $(65.6 \%$ vs $71.1 \%$; $P=0.0380$ ) (Fig. 3). Infliximab/IBD had a significantly higher percentage of persistent patients than infliximab/RA ( $71.1 \%$ vs $64.0 \%$; $P=0.0119)$. Similarly, infliximab/RA had a significantly higher percentage of persistent patients than tofacitinib/RA (64.0\% vs $54.9 \%$; $P=0.0026)$, again highlighting the potential impact of route of administration on treatment persistence within the same disease state.

Patients on vedolizumab had a $28 \%$ lower risk of discontinuation (hazard ratio [HR] 0.7; 95\% CI $0.6-0.9$ ) and $65 \%$ increased odds of persistence (OR 1.7; 95\% CI 1.3-2.1) compared with patients on tofacitinib (Table S3). After indirect adjustment for disease state, there was only a $3.6 \%$ difference in the proportion of persistent patients between those initiating vedolizumab compared with tofacitinib $(P=0.4640)$.

When a more restrictive definition of discontinuation was used that classified any interruption of at least 0.5 times the prior day's supply (beginning from the end of the last supply) as a gap in therapy, the proportions of patients persistent at 12 months of follow-up dropped overall, and times to discontinuation were shorter; however, results of the group comparisons and overall trends remained unchanged (Fig. S2; Table S3).

Because both biologic-naïve and biologicexperienced patients were included in the primary analysis, a post hoc analysis of persistence among patients who were biologic naïve was conducted; the results of the post hoc analysis were similar to the primary analysis (Fig. S3). Time to treatment discontinuation in biologicnaive patients was also consistent with the overall population (time to $25 \%$ discontinuation 201 days [vedolizumab/IBD] vs 136 days [tofacitinib/RA]; $P=0.0377$ ) (Fig. S4).

\section{DISCUSSION}

This study provides perspective on the potential implications of route and frequency of dosing on adherence and persistence for treatment of IBD (CD and UC). Currently, biologics 


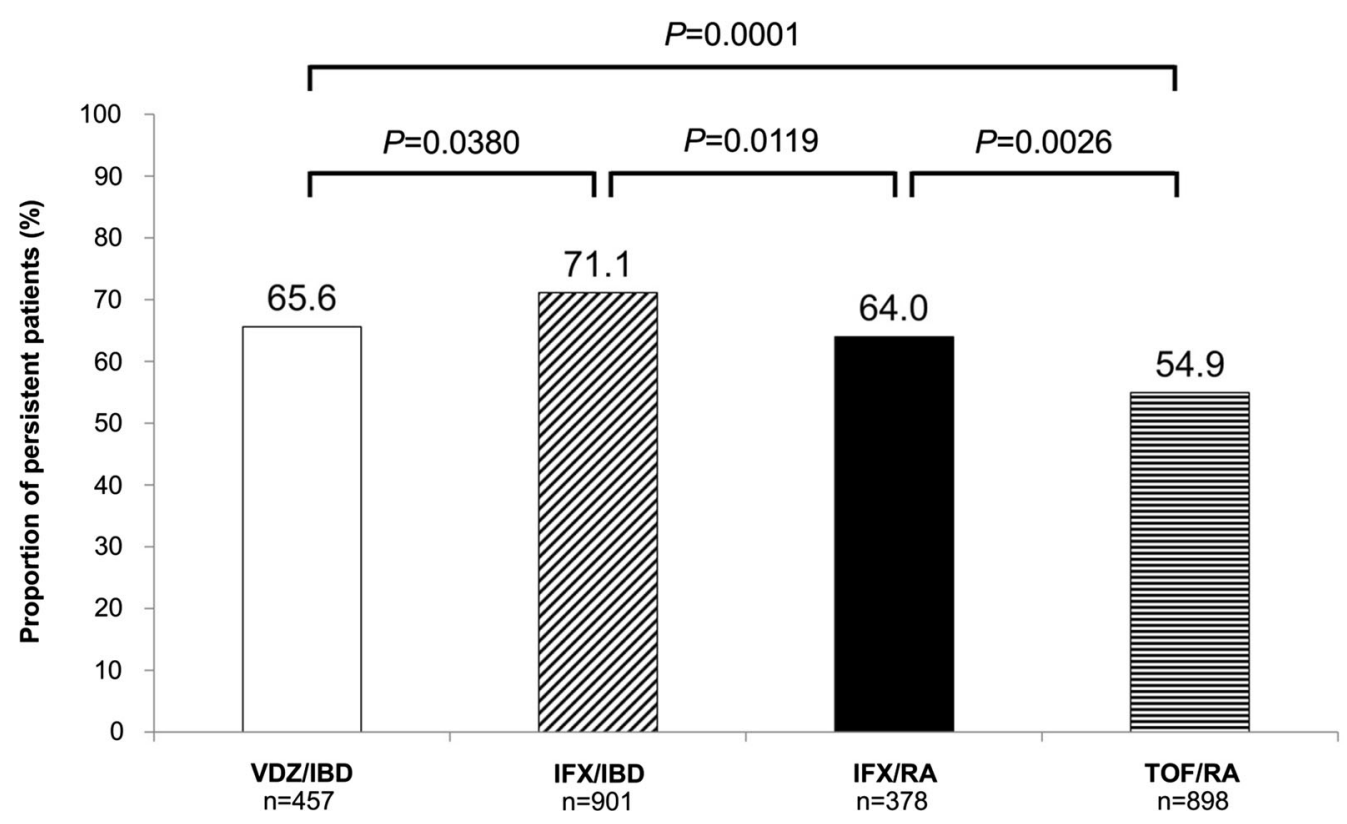

Fig. 3 Proportion of persistent patients initiating vedolizumab, infliximab, or tofacitinib after 12 months of follow-up. Nonpersistence was defined as gap in therapy $\geq 1.5$ times the prior day's supply, beginning from the end

(subcutaneous or intravenous) are the mainstay of treatment for these indications. New therapies with novel routes of administration are being tested in patients with IBD. Approval of tofacitinib in May 2018 for the treatment of UC offers patients and clinicians an option to use an oral drug formulation, which has notable perceived benefits, such as increased convenience [16, 17]. Such perceived advantages should be weighed against the risk for lower adherence [11]. In a single-center, retrospective cohort study, facility-administered biologics were found to be independently associated with higher adherence than self-administered biologics, with highest adherence reported for vedolizumab (83\%) compared with infliximab (70\%), adalimumab (57\%), and certolizumab pegol (50\%) [18]. Although adherence data in a given disease accumulate over extended periods, it would be useful for practitioners to gain early insights into potential issues that could have negative long-term effects.

In this study, we assessed adherence to and persistence on (1) vedolizumab among patients of the last supply. IBD inflammatory bowel disease, IFX infliximab, RA rheumatoid arthritis, TOF tofacitinib, VDZ vedolizumab

with IBD, (2) tofacitinib among patients with RA, and (3) infliximab among patients with either IBD or RA. There have been previous comparisons of adherence to medications across rheumatologic disease states $[19,20]$; however, in acknowledgment of the disease state differences, any comparison of adherence or persistence in this study among patients with IBD compared with RA is indirect.

Overall, our results indicate that by indirect comparison, adherence and persistence were consistently higher with vedolizumab than with tofacitinib, even after adjustment for disease state differences. Adherence to and persistence on an intravenous treatment (infliximab) were higher than with oral treatment (tofacitinib) in RA, which suggests that there might be similar differences in adherence to IBD therapies by route of administration. The less supervised setting associated with oral medication could be a contributing factor to lower adherence rates than intravenous therapy administered in an infusion center [11]. Increased supervision coupled with increased interaction with healthcare providers at infusion centers 
might be positive factors in promoting adherence [11]. Dosing schedule complexity has also been associated with poor adherence to oral medications [1]. In fact, once-daily dosing was associated with significantly better adherence rates than twice-daily dosing [21].

Factors other than route of administration could have an impact on medication adherence [1]. Effectiveness and tolerability of treatment are also important factors in patient persistence to and adherence on treatment [3].

The efficacy and safety of vedolizumab have been supported by the results of the GEMINI clinical studies and real-world data, as published previously [22-27]. Vedolizumab has been shown to be well tolerated in a clinical trial setting, with only a slightly higher risk of infections and nasopharyngitis than placebo [22]. In two real-world studies in patients with UC and CD, persistence rates with vedolizumab ranged from $52 \%$ to $88 \%$ in UC and from $40 \%$ to $67 \%$ in $C D$ at week $54[24,25]$. In the Swedish National Quality Registry for IBD (SWIBREG), the persistence rates for 6 and 12 months were $76 \%$ and $65 \%$ for UC and $75 \%$ and $61 \%$ for $\mathrm{CD}$, respectively [27].

Tofacitinib was investigated as an induction therapy in UC in two phase 3 clinical trials (OCTAVE-1 and -2) [28]. Results from these studies demonstrated that tofacitinib achieved a therapeutic benefit. Although the safety profile of tofacitinib did not differ from placebo in the short (8-week) UC studies, in the OCTAVE Sustain trial, rates of overall infection and herpes zoster infection were higher with tofacitinib than with placebo [28], and across all three trials, adjudicated non-melanoma skin cancer and adjudicated cardiovascular events were higher with tofacitinib. Recently, the FDA issued a safety alert about a potential increased risk of blood clots in the lungs and death with a 10-mg twice-daily dose of tofacitinib based on safety trial data conducted in RA [29].

\section{LIMITATIONS}

Consistent evidence in this analysis suggests that there are strong differences in adherence to and persistence on medications between the disease states studied (IBD and RA). Although infliximab was used as a validation comparison and the difference in adherence among patients taking infliximab for IBD compared with infliximab for RA was adjusted, these comparisons are indirect. The results of this study might not be generalizable to uninsured patients, Medicaid beneficiaries, other Medicare beneficiaries, or patients with other types of insurance not represented in the Truven MarketScan ${ }^{\circledR}$ database. Our study was limited by the inherent limitations of claims database studies, including potential data entry errors, miscoding or billing, duplicate claims, or payer influences. This study used prescription fill information, a proxy measure of medication-taking behavior, to measure medication adherence and persistence for tofacitinib; whether patients actually took the medication as directed after dispensing cannot be known. This study did not adjust for pill burden, but attempted to adjust for medication regimen complexity using prescription burden instead; prescription burden was not statistically significant in our model and was therefore dropped for parsimony. Additionally, there are several reasons for discontinuation that pertain to each disease that could explain the higher discontinuation seen in the tofacitinib group. Our study was also limited by the lack of data surrounding disease activity status, which may contribute to medication adherence and persistence. Patients with severe disease are often more adherent to medications and are more likely to be prescribed infusion-based medications than patients with milder symptoms [5]. Finally, adherence and persistence were determined in the overall group of patients with IBD because conducting these analyses in patients with UC or CD would have limited the sample size and the robustness of the study.

\section{CONCLUSIONS}

We describe a novel adjustment method for interdisease treatment differences using infliximab treatment patterns to bridge intrinsic differences between IBD and RA. Study results provide important insights into understanding the impact of route of administration on 
adherence and persistence in IBD. Adherence and persistence were consistently higher for infusion therapy than for oral medications when directly comparing infliximab to tofacitinib in RA and indirectly comparing vedolizumab in IBD to tofacitinib in RA. Lower adherence to and persistence on oral treatment could affect overall efficacy in the management of IBD. Future work is needed to investigate the association between route of administration, medication utilization, and outcomes as new formulations become available.

\section{ACKNOWLEDGEMENTS}

Funding. The authors received financial support from Takeda Pharmaceuticals U.S.A., Inc. for the research, authorship, study support, and all publication costs associated with this article including Rapid Service Fee and Open Access charges. All authors had full access to all of the data in this study and take complete responsibility for the integrity of the data and accuracy of the data analysis.

Medical Writing, Editorial and Other Assistance. The authors thank Yaping Wang, Charlie Cao, and Michelle Luo (Takeda Pharmaceuticals U.S.A., Inc.) for help with the analyses. Medical writing assistance was provided by Sangeeta De, PhD, and Reem Berro, $\mathrm{PhD}$, of inVentiv Medical Communications, LLC, a Syneos Health ${ }^{\mathrm{TM}}$ group company, and funded by Takeda Pharmaceuticals U.S.A., Inc.

Authorship. All named authors meet the International Committee of Medical Journal Editors (ICMJE) criteria for authorship for this article, take full responsibility for the integrity of the work as a whole, and have given their approval for this version to be published.

Prior Presentation. Some data included in this manuscript were presented at the 2018 European Crohn's and Colitis Organisation and Digestive Disease Week congresses.
Disclosures. Sunanda Kane has served as a consultant for AbbVie, Merck, Samsung Bioepis, Seres Therapeutics, and Spherix Health; has received research funding from UCB; and is a member of the GI Specialty Board of the American Board of Internal Medicine. Kellyn Moran is an employee of Takeda Pharmaceuticals U.S.A., Inc. Kyle Null is an employee of Takeda Pharmaceuticals U.S.A., Inc. Zhongwen Huang is an employee of Takeda Pharmaceuticals U.S.A., Inc. Trevor Lissoos was an employee of Takeda Pharmaceuticals U.S.A., Inc. when the study was being conducted and is now an employee of Eli Lilly \& Company.

Compliance with Ethics Guidelines. This study only involved the use of anonymized electronic healthcare records, and researchers did not have access to named or identifiable patient information. As such, this study does not meet the definition of human subject research as defined in federal regulations 45 CFR 46.102. The study was therefore exempt from institutional review board review.

Data Availability. All data used in this study were retrieved from the Truven MarketScan $^{\circledR}$ database, which is part of the IBM Watson Health ${ }^{\mathrm{TM}}$ business.

Open Access. This article is distributed under the terms of the Creative Commons Attribution-NonCommercial 4.0 International License (http://creativecommons.org/licenses/ by-nc/4.0/), which permits any noncommercial use, distribution, and reproduction in any medium, provided you give appropriate credit to the original author(s) and the source, provide a link to the Creative Commons license, and indicate if changes were made.

\section{REFERENCES}

1. Herman ML, Kane SV. Treatment nonadherence in inflammatory bowel disease: identification, scope, and management strategies. Inflamm Bowel Dis. 2015;21(12):2979-84. https://doi.org/10.1097/MIB. 0000000000000581 . 
2. Li P, Blum MA, Von Feldt J, Hennessy S, Doshi JA. Adherence, discontinuation, and switching of biologic therapies in Medicaid enrollees with rheumatoid arthritis. Value Health. 2010;13(6):805-12. https://doi.org/10.1111/j.1524-4733.2010.00764.x.

3. Morrison VL, Holmes EA, Parveen S, et al. Predictors of self-reported adherence to antihypertensive medicines: a multinational, cross-sectional survey. Value Health. 2015;18(2):206-16. https://doi.org/ 10.1016/j.jval.2014.12.013.

4. Rannanheimo PK, Tiittanen P, Hartikainen J, et al. Impact of statin adherence on cardiovascular morbidity and all-cause mortality in the primary prevention of cardiovascular disease: a populationbased cohort study in Finland. Value Health. 2015;18(6):896-905. https://doi.org/10.1016/j.jval. 2015.06.002.

5. Lakatos PL. Prevalence, predictors, and clinical consequences of medical adherence in IBD: how to improve it? World J Gastroenterol. 2009;15(34): 4234-9.

6. Lopez A, Billioud V, Peyrin-Biroulet C, Peyrin-Biroulet L. Adherence to anti-TNF therapy in inflammatory bowel diseases: a systematic review. Inflamm Bowel Dis. 2013;19(7):1528-33. https:// doi.org/10.1097/MIB.0b013e31828132cb.

7. Panaccione R, Rutgeerts P, Sandborn WJ, Feagan B, Schreiber S, Ghosh S. Review article: treatment algorithms to maximize remission and minimize corticosteroid dependence in patients with inflammatory bowel disease. Aliment Pharmacol Ther. 2008;28(6):674-88. https://doi.org/10.1111/j.13652036.2008.03753.x.

8. Jackson CA, Clatworthy J, Robinson A, Horne R. Factors associated with non-adherence to oral medication for inflammatory bowel disease: a systematic review. Am J Gastroenterol. 2010;105(3):525-39. https://doi.org/10.1038/ajg. 2009.685 .

9. Bressler B, Haraoui B, Keystone E, Sette A. Optimizing use of tumor necrosis factor inhibitors in the management of immune-mediated inflammatory diseases. J Rheumatol Suppl. 2010;85:40-52. https://doi.org/10.3899/jrheum.091464.

10. Vangeli E, Bakhshi S, Baker A, et al. A systematic review of factors associated with non-adherence to treatment for immune-mediated inflammatory diseases. Adv Ther. 2015;32(11):983-1028. https:// doi.org/10.1007/s12325-015-0256-7.

11. Seal BS, Anderson S, Shermock KM. Factors associated with adherence rates for oral and intravenous anticancer therapy in commercially insured patients with metastatic colon cancer. J Manag Care
Spec Pharm. 2016;22(3):227-35. https://doi.org/10. 18553/jmcp.2016.22.3.227.

12. Entyvio $^{\circledR}$ (vedolizumab) [prescribing information]. Deerfield: Takeda Pharmaceuticals America; 2014.

13. Xeljanz ${ }^{\circledR} \quad$ (tofacitinib) [prescribing information]. New York: Pfizer; 2017.

14. US Food and Drug Administration. FDA approves new treatment for moderately to severely active ulcerative colitis [press release]. Silver Spring: US Food and Drug Administration; 2018. https://www. fda.gov/news-events/press-announcements/fda-app roves-new-treatment-moderately-severely-active-ul cerative-colitis. Accessed 1 May 2019.

15. Remicade ${ }^{\circledR}$ (infliximab) [prescribing information]. Horsham: Janssen Biotech, Pfizer; 2013.

16. Alten R, Kruger $\mathrm{K}$, Rellecke J, et al. Examining patient preferences in the treatment of rheumatoid arthritis using a discrete-choice approach. Patient Prefer Adherence. 2016;10:2217-28.

17. Eek D, Krohe M, Mazar I, et al. Patient-reported preferences for oral versus intravenous administration for the treatment of cancer: a review of the literature. Patient Prefer Adherence. 2016;10:1609-21. https://doi.org/10.2147/PPA. S106629.

18. Wentworth BJ, Buerlein RCD, Tuskey AG, Overby MA, Smolkin ME, Behm BW. Nonadherence to biologic therapies in inflammatory bowel disease. Inflamm Bowel Dis. 2018;24(9):2053-61. https:// doi.org/10.1093/ibd/izy102.

19. Howe A, Eyck LT, Dufour R, Shah N, Harrison DJ. Treatment patterns and annual drug costs of biologic therapies across indications from the Humana commercial database. J Manag Care Spec Pharm. 2014;20(12):1236-44.

20. Degli Esposti L, Sangiorgi D, Perrone V, et al. Adherence and resource use among patients treated with biologic drugs: findings from BEETLE study. Clinicoecon Outcomes Res. 2014;6:401-7. https:// doi.org/10.2147/CEOR.S66338.

21. Srivastava K, Arora A, Kataria A, Cappelleri JC, Sadosky A, Peterson AM. Impact of reducing dosing frequency on adherence to oral therapies: a literature review and meta-analysis. Patient Prefer Adherence. 2013;7:419-34. https://doi.org/10. 2147/PPA.S44646.

22. Sandborn WJ, Feagan BG, Rutgeerts P, et al. Vedolizumab as induction and maintenance therapy for Crohn's disease. N Engl J Med. 2013;369(8):711-21. https://doi.org/10.1056/NEJMoa1215739. 
23. Ylisaukko-Oja T, Aaltonen J, Nuutinen $\mathrm{H}$, et al. High treatment persistence rate and significant endoscopic healing among real-life patients treated with vedolizumab: a Finnish Nationwide Inflammatory Bowel Disease Cohort Study (FINVEDO). Scand J Gastroenterol. 2018;53(2):158-67. https:// doi.org/10.1080/00365521.2017.1416160.

24. Allegretti JR, Barnes EL, Stevens B, et al. Predictors of clinical response and remission at 1 year among a multicenter cohort of patients with inflammatory bowel disease treated with vedolizumab. Dig Dis Sci. 2017;62(6):1590-6. https://doi.org/10.1007/ s10620-017-4549-3.

25. Stallmach A, Langbein C, Atreya R, et al. Vedolizumab provides clinical benefit over 1 year in patients with active inflammatory bowel disease: a prospective multicenter observational study. Aliment Pharmacol Ther. 2016;44(11-12):1199-212. https://doi.org/10.1111/apt.13813.

26. Vivio EE, Kanuri N, Gilbertsen JJ, et al. Vedolizumab effectiveness and safety over the first year of use in an IBD clinical practice. J Crohns Colitis.
2016;10(4):402-9. https://doi.org/10.1093/ecco-jcc/ jjv226.

27. Eriksson C, Marsal J, Bergemalm D, et al. Long-term effectiveness of vedolizumab in inflammatory bowel disease: a national study based on the Swedish National Quality Registry for Inflammatory Bowel Disease (SWIBREG). Scand J Gastroenterol. 2017;52(6-7):722-9. https://doi.org/10.1080/0036 5521.2017 .1304987$.

28. Sandborn WJ, Su C, Sands BE, et al. Tofacitinib as induction and maintenance therapy for ulcerative colitis. N Engl J Med. 2017;376(18):1723-36. https://doi.org/10.1056/nejmoa1606910.

29. US Food and Drug Administration. Safety trial finds risk of blood clots in the lungs and death with higher dose of tofacitinib (Xeljanz, Xeljanz XR) in rheumatoid arthritis patients; FDA to investigate. https://www.fda.gov/drugs/drug-safety-and-availabi lity/safety-trial-finds-risk-blood-clots-lungs-and-dea th-higher-dose-tofacitinib-xeljanz-xeljanz-xr. Published February 25, 2019. Accessed 1 May 2019. 